\title{
Comparison of the Stability of Different Arthroscopic Tendon Fixation Techniques in Dynamic Tests
}

\author{
W. Anderl*, R. Reihsner ${ }^{\dagger}$, P. R. Heuberer*, B. Kriegleder* and J. Eberhardsteiner ${ }^{\dagger}$ \\ *Orthopaedic Department, St. Vincent Hospital, Vienna, Austria \\ †Institute for Mechanics of Materials and Structures, Vienna University of Technology, Austria
}

\begin{abstract}
In studies concerning the stability of surgical tendon fixation techniques, a large variety of test configurations is found resulting in a broad scatter of results. The goal of this study was to establish a reliable test protocol for the investigation of the stability of repair techniques. At 88 fresh bovine shoulders, four different suture configurations were tested: simple stitches, mattress sutures, modified Mason-Allen configuration and double-row configuration. The difference in response to load cycles with native samples and that after repair was recorded. Bioresorbable anchors were used for tendon fixation, in a second run tendons were affixed to metal plates, and with a third run fixation stability was monitored with a video camera. The double-row technique was the most stable one with the lowest values of compliance at all selected levels of gap formation followed by the mattress suture configuration. The simple stitch configuration and the modified Mason-Allen techniques were the most compliant techniques. By establishing this test protocol, we achieved a very accurate method of measurement (accuracy of $2 \mu \mathrm{m}$ ) with as few external influences as possible. The great advantage is that the proposed protocol allows the measurement of the mechanical quality of the suture fixation without an external device such as calipers.
\end{abstract}

KEY WORDS: arthroscopic rotator cuff repair, arthroscopic suture configuration, dynamic biomechanical testing

\section{Introduction}

The prevalence of rotator cuff tears in the population has been estimated from 32 to $80 \%$ depending on the age of the patient [1-5]. Therefore, it is one of the most common surgical procedures in the shoulder. The goal of surgery is an early functional mobilization of the patient. The most critical phase for success or failure of a rotator cuff repair is the early postoperative phase while load transfer is entirely carried through the fixation. As re-tear rates after arthroscopic cuff repair of large and massive tears differ from 22 to over $90 \%$ a lot of biomechanical studies were performed to assess the best fixation configuration in vitro [6-12]. Mechanisms of implant failure include anchor pullout, knot slippage, suture breakage or pullout. The most probable source of failure is the tendon-suture interface [13-15].

There are a lot of studies with plenty of different results. A large variety of test configurations do not allow an easy interpretation and comparison of results in different studies. This variety comprises the donors of samples (human or bovine), the storage of samples until test (at room temperature or frozen at $-20{ }^{\circ} \mathrm{C}$ ), the method to clamp samples in the test machine, the method to create the defect (crescentshaped or total cutoff), the loading scheme, the way to measure the gap between tendon and bone, the number of samples and last but not least the evaluation of data.

There are studies where fresh-frozen human shoulders [16-21] were used, whereas others [22-24] used fresh-frozen or fresh immature [25] bovine shoulders, also pig tendons [26] or fresh sheep tendons [22] were used for the tests. Some authors detached the tendon insertion completely [22, 27], others formed a crescent-shaped defect $[16,19,23-$ 25]. In one investigation [28], the bone was embedded in a mould and a low temperature melting alloy, in another one the humerus was fixed in a metal cylinder with K-wires [21] or in epoxy resin [25]. In some studies, the tendon was fixed proximally with a cryo-clamp $[19,29]$, others attached the free end of the tendon via a nylon strap [16, 22] to the test apparatus. The studies varied also in the pretension which was applied before the test started 
from 5 to $40 \mathrm{~N}[18,19]$ or with a pre-conditioning phase of $20 \mathrm{~min}$ at a load level of $20 \mathrm{~N}$ [21], in the speed of cyclic loading varying from 5 [17] to $33 \mathrm{~mm} \mathrm{~s}^{-1}$ [16] or, in the form of applying the cyclic load continuously $[26,27]$ or reaching a plateau, performing some cycles, increasing the load, performing again some cycles and so on [16, 19]. The final load also differed from 90 to $180 \mathrm{~N}[16$, 25]. The gap formations were measured with a metric ruler [16], digital calipers [24, 25], markers $[17,18,29]$, an extensometer [27] or the information was not provided in the study [21]. The amount of cyclic loading was differing from 50 [18] to 2500 cycles $[16,24]$. Some recent biomechanical studies demonstrated that the mechanical strength of a double-row cuff repair is superior to a single-row repair [17-19], other studies showed no difference in results $[20,25]$. In single-row repair, some studies say that the modified Mason-Allen suture configuration performs best [23], other studies show that the massive single stitch does better [29], again others did not find any difference [24].

This is only a compendium and not exhaustive but from this literature review a considerable lack of comparable test configurations and test results is realized. The aim of this study was to compare the biomechanical stabilities of different arthroscopic suture techniques. Two different methods to measure gap formation were applied and also the influence of the usage of anchors or not were studied.

The most prominent requirements for the experimental procedure are:
- The biomechanical status of the tendon-bone biomaterial should not be altered by the experimental procedure (e.g. storage temperature until test, clamping procedures, like epoxy resin, low temperature melting alloys).

- Natural loading situation should be simulated by the tests.

- The compliance of the tendon-bone joint should be extracted from the whole system (e.g. measuring exactly the gap formation, if possible, or by additional recording of the biomechanical properties of the biomaterials used).

- The clamping should be saved (no slippage effect, e.g. via the attachment of nylon straps to the free tendon end) and it also should not influence the anchor-suture system (e.g. temperature increase when using epoxy resin).

- The load should be transmitted to the anchorsuture system only (e.g. when using crescentshaped defects, the load will partially transfer through the remaining natural tendon-bone joint).

- $\quad$ Averaging procedures for data evaluation should be used (e.g. least mean square fit).

The notion 'rotator cuff' is illustrated by Figure $1 \mathrm{~A}$ (taken from a textbook) showing a human shoulder with a rupture of the tendon of the rotator cuff. In this study, a bovine rotator cuff was used for the different investigations The tendon under consideration (analogous to the human one shown in the figure) was completely dissected from the bone and

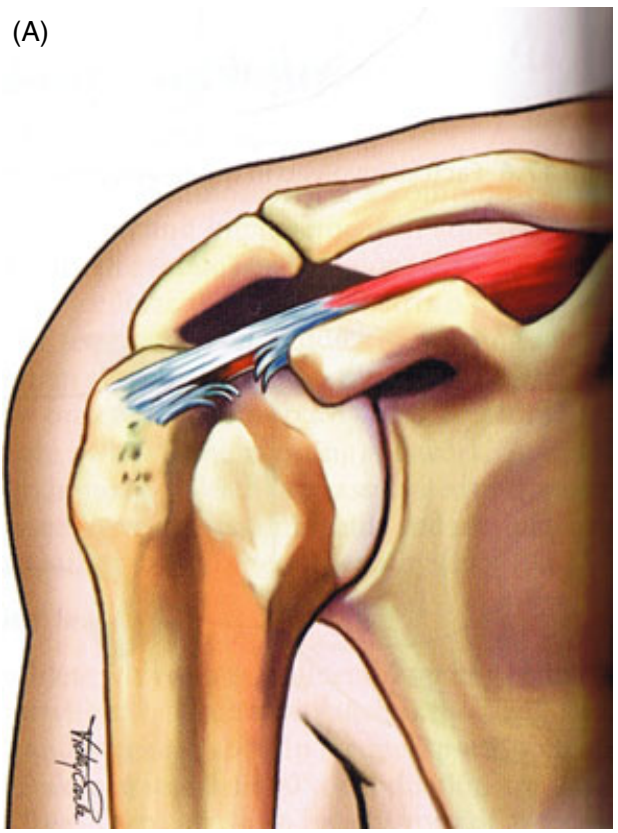

(B)

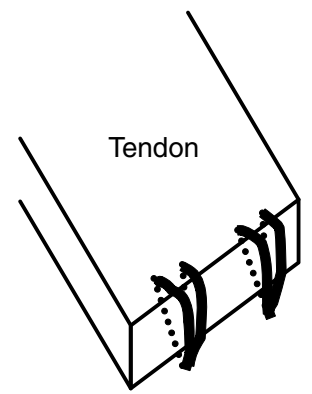

4 single loops along the fiber direction of the tendon (S)

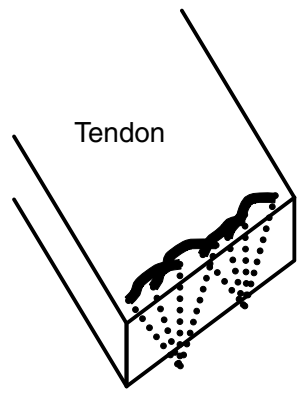

4 loop across the fiber direction of the tendon (M)

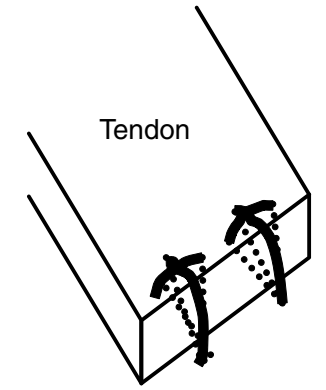

The methods combined (Mason Allen, MA)

Figure I: (A) Rupture of a rotator cuff tendon. (B) Sketch of the suture configurations used in the study. The sutures are affixed to the bone via 2 anchors. The double row technique (D) may be thought as a combination of the mattress (M) technique and the MA technique using a third anchor 
refixed with different suture-anchor techniques as shown in Figure 1B. To implant the bioresorbable anchors, a screw tap was used and two anchors for four commonly used suturing techniques were screwed into the bone. Each anchor is equipped with two sutures which were then tied to give the different configurations. The manipulations were carried out with medical instruments as used in surgery.

\section{Materials and Methods}

\section{Specimens}

Eighty-eight bovine shoulders (Figure 2A,B) were used for the investigation. The samples were brought from the abattoir in the morning. Pre-tests (about $30 \mathrm{~min}$ ) were performed to obtain the (bio)mechanical properties of the intact samples. The positions of the clamps (Figure 3 ) for bone and tendon were marked to give the same clamping conditions for the main test. The samples were stored in the refrigerator $\left(4{ }^{\circ} \mathrm{C}\right)$ until surgical manipulations. The tendon was detached completely from the bone. It was dissected to give a width of $20 \mathrm{~mm}$ like in other studies [16]. This was performed in the early afternoon of the same day. Then the first test was conducted parallel to the surgical procedure with the other samples, which were stored in the refrigerator after surgery until test with a maximum period of storage of $24 \mathrm{~h}$. The test period was about $4 \mathrm{~h}$. Freezing the samples at $-20^{\circ} \mathrm{C}[16,22-24]$ was avoided as it may irreversibly affect the biomechanical quality of the samples and also that of the suture-anchor system.

\section{Defect preparation and suture configuration}

The investigation was done in three parts and with four suture configurations. In part 1 of the study, the tendons $(n=31)$ were attached to the bone via anchors and different suture techniques. In parts 2 and 3 , the tendons ( $n=25$ and 32, respectively) were attached to metal plates by the same suture techniques via metal wires (Figure 4 ). As to the suture configurations, two anchors were used with (i) four simple stitches (S), (ii) four mattress sutures (M) and (iii) two modified Mason-Allen sutures (MA) according to Scheibel [30]. Suture configuration 4 was our double-row technique (D) consisting of two medial placed anchors with mattress sutures and one lateral anchor with two single stitches running across the mattress sutures was performed to fix the cuff. The anchors (Arthrex Corkscrew bioresorbable $5.5 \mathrm{~mm}$ )

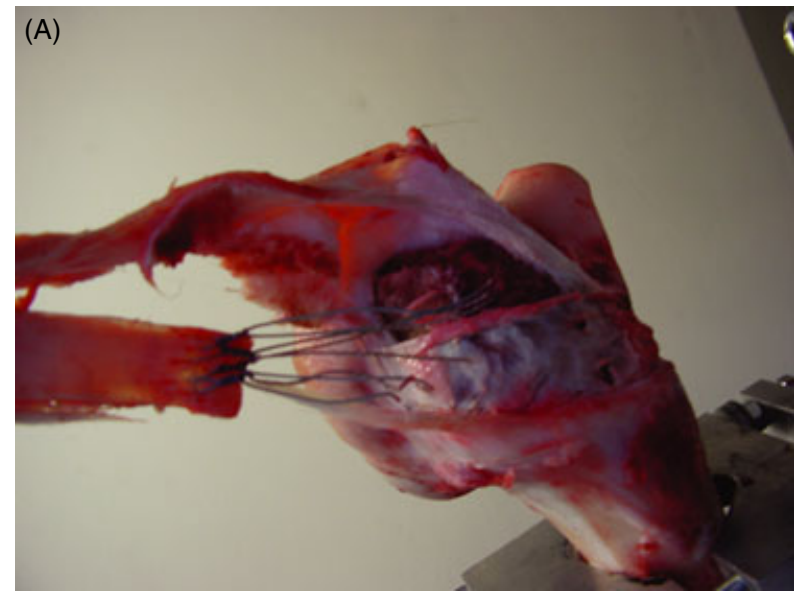

Typical bone-tendon sample

(B)

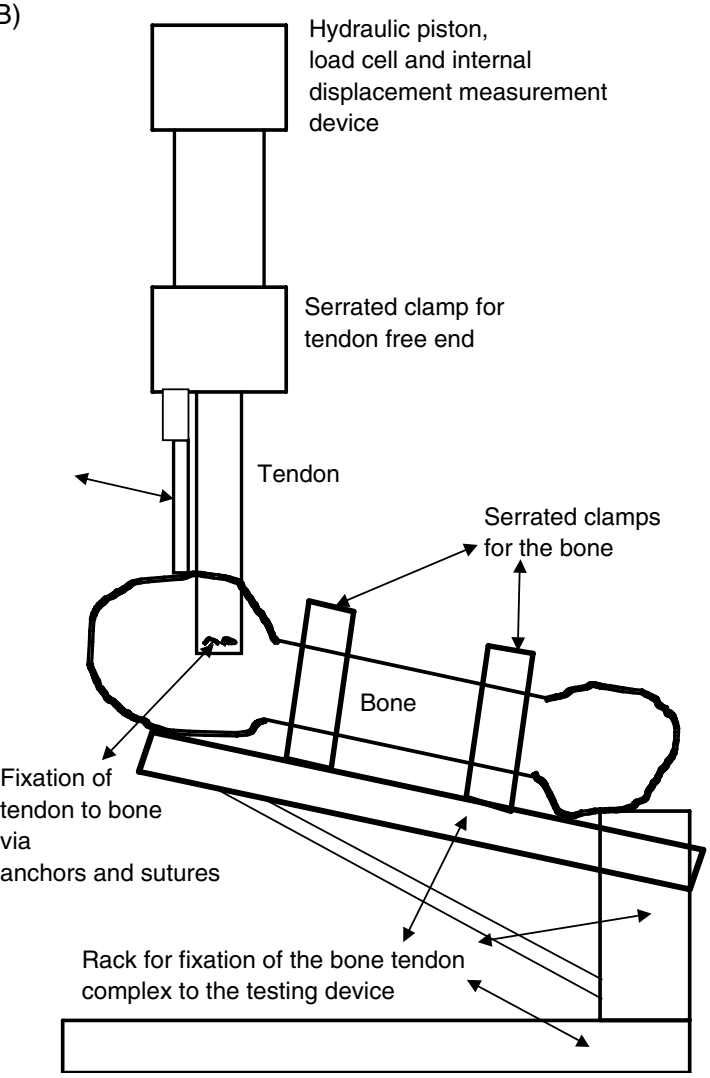

Figure 2: (A) Typical bone-tendon sample. (B) Line diagram of the set-up including a sketch of the sample (see also Figure 1A) and the metal rack and clamps

were inserted into the bone at an angle of $45^{\circ}$ and all sutures were tied in an arthroscopic way with a Nicky's knot [31].

These four suture configurations have been used in part 1 of the investigation (fixation to bone) and also in part 2 (fixation to the metal plate). The suture configurations employed in part 2 resemble that used in part 1 . In part 2 , metal wires laterally plugged in the metal plate play the role of the anchor's eyelet. In part 3 of the study suture, the configurations 1, 2 and 3 were employed with two sutures for configuration 1 


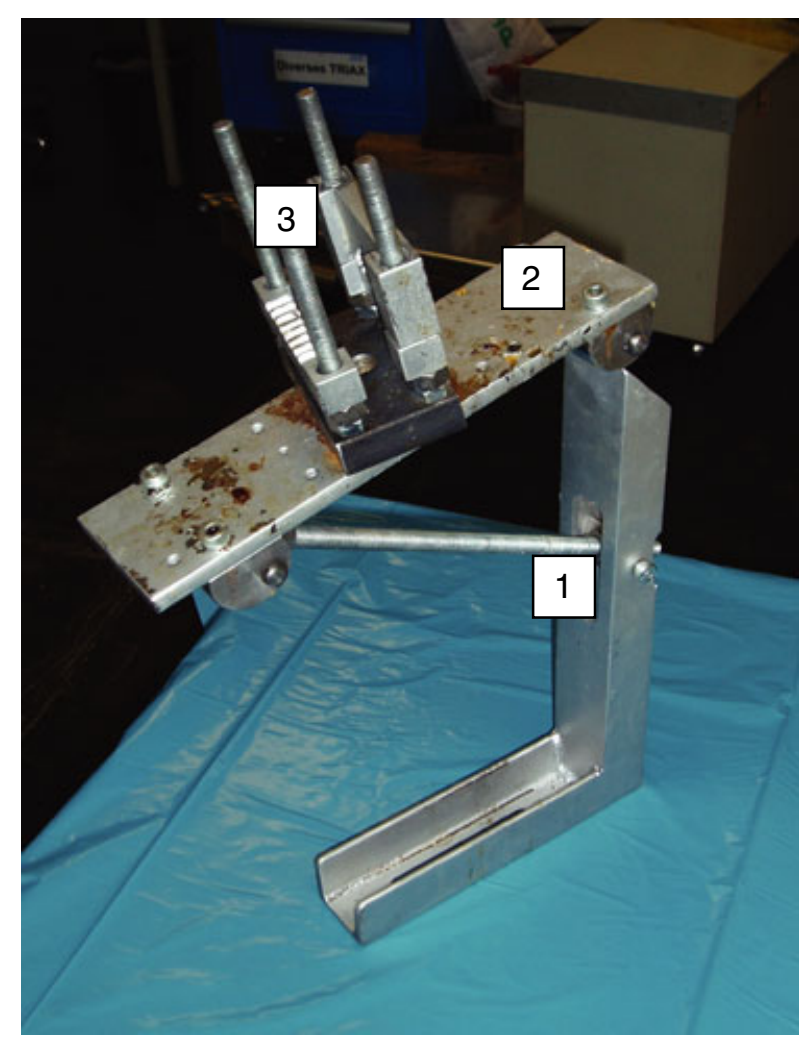

Figure 3: Device for the fixation of the bone-tendon complex, which is mounted to the base plate of a hydraulic loading machine. The device consists of a metal rack (1), a swivelmounted platform (2) and a serrated clamp for the bone (3)

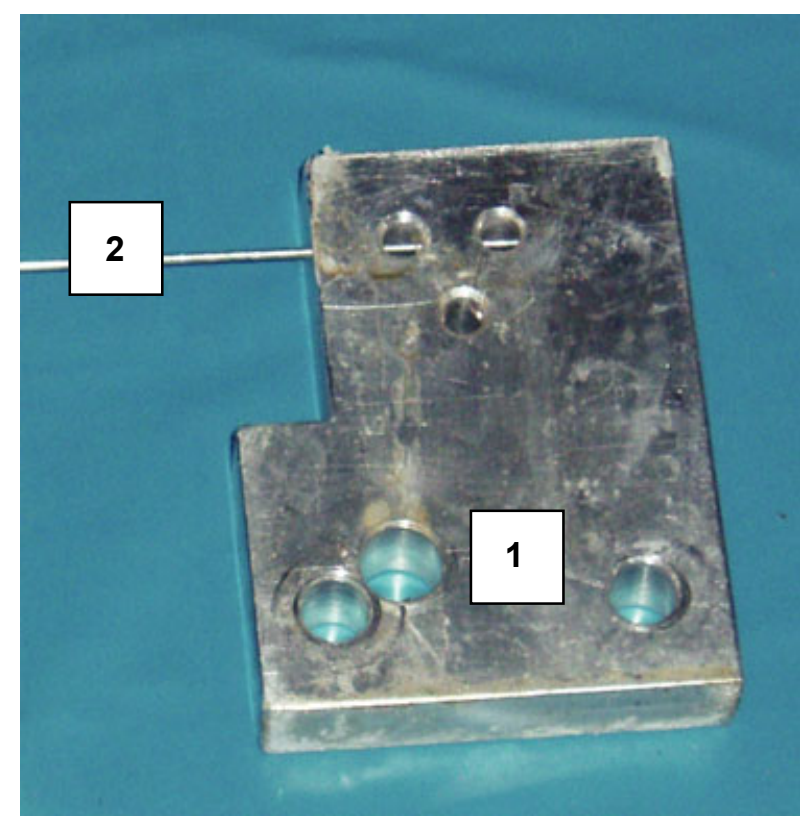

Figure 4: Metal plate (1) for the fixation of the tendon via different suture techniques using a metal wire of $0.7 \mathrm{~mm}$ diameter (2)

and 2, and one MA-technique for configuration 3, i.e. half the number of 'threads' for all configurations in part 3.

\section{Test strategy}

As we are concerned with a compound of materials with different compliances (bone, tendon, and the joint between tendon and bone), the test method should be selected carefully to separate clearly the contributions of the different elements in the compound. In this study, cyclic loading between two levels of load was performed. As mechanical response the elastic elongation of the tendon will also cycle between corresponding levels superimposed by creep showing an exponential increase of these levels. The creep behaviour of the tendons as a feature of viscoelasticity was recorded by means of a pre-test before application of the distinct suture systems. After re-fixation of the tendons to the bone, there is an additional creep as a result of the weakening of the suture system by load cycling. There are, of course, individual variations in the biomechanical qualities of the specimens used. Hence, to each test a pre-test with the intact bone-tendon complex was conducted. The creep behaviour was fitted by a function $\delta$ [Equation (1), elongation $\delta$ of the tendon versus cycle number] that allows an extrapolation (of the pre-test or of the main test if failure is not reached within the maximum number of cycles) to the final value of displacement corresponding to the load used. The fit with function $\delta$ results in the determination of the parameters $a, b, \tau$ and $\lambda$ [Equation (1)]. The test with the re-attached tendon will result in another elongation-cycle number relationship $\delta$. To determine the cycle number to reach a chosen gap the curve of the pre-test was shifted by that amount of gap and intersected with the measured curve of the re-attached tendon. That way gap formation was measured irrespective of the individual viscoelastic properties of tendons. The method cannot address slippage in the clamp (free tendon end). Therefore, in an extra study (part 3), markers (pins) were attached to the tendon, a ruler attached to the metal plate and the gap formation monitored with a video film. The cycle number to reach a chosen gap level was counted. Within this part of the study, three suture systems ( $\mathrm{S}, \mathrm{M}$ and $\mathrm{MA}$ ) were applied, and in all cases an excellent correlation between the two gap measurement methods was obtained $(r=98.6 \%)$. However, one can only reasonably use the video method to count 100 or perhaps 200 cycles. It will not properly work with very stable suture configurations, as in part 1 or 2 of the present study, with 2000 or more cycles needed to reach a gap of $5 \mathrm{~mm}$. The method to perform pre-tests has also the advantage that individual variations in tendon length are compensated. The positions of the clamps were marked on bone and 
tendon with a water-proof marker for an equal clamping in the main test after the surgical procedure.

\section{Loading scheme and experimental procedure}

Cyclic loading was performed between the levels of 10 and $180 \mathrm{~N}$, with displacement-controlled ramps of $\pm 33 \mathrm{~mm} \mathrm{~s}^{-1}$. The period of each cycle was $5 \mathrm{~s}$ in total, approx. $2.5 \mathrm{~s}$ for 10 and $180 \mathrm{~N}$, each. The tests were stopped at a maximum number of cycles of 2500 or when reaching a gap of $10 \mathrm{~mm}$. This loading scheme follows that of Burkhart [16] who stressed, that the chosen speed of loading and the load plateau is similar to that of normal daily human activity and the number of cycles corresponds to that of a healing phase [32]. The displacements were measured with the internal position measurement device of the testing equipment (with an accuracy of $2 \mu \mathrm{m}$ ). Also the bending of the bone was measured with an external device (dial gauge, accuracy $2 \mu \mathrm{m}$ ). The built-in load cell has a maximum load of $10 \mathrm{kN}$ at an accuracy of $1 \mathrm{~N}$. An hydraulic equipment was used for the tests, with electronic support and computer assistance (Walter \& Bai, Lohningen, Switzerland). A serrated clamp (Figure 3) was used to attach the bone to the mounting plate of the test equipment. The direction of the bone was at an angle to the mounting plate (Figure 3 ) such that the tendon could be loaded in its natural line of tensile loading. This angle could be adjusted individually for each specimen by a proper construction of the metal rack on which the serrated clamps were mounted (Figure 3) for fixation of the bone. The rate of data recording was $100 \mathrm{~s}^{-1}$ such that the response to the loading ramps as well as the time-dependent (rheological) behaviour at the load plateaus could be measured accurately.

We preferred to use a mechanical way of clamping the bone instead of a chemical one like embedding it in a mould with epoxy resin $[24,25]$ as the hardening procedure of the epoxy resin might cause a temperature above $40{ }^{\circ} \mathrm{C}$, which may irreversibly damage the bone. The free ends of the tendons were attached to the hydraulic piston by means of a serrated clamp as used by others [24]. This method was reported to give a save grip (no slippage effects) and was preferred to the use of a cryo-clamp [19, 23, 27] also for reasons of temperature effects or nylon strap attachment [16, 22] as it is not safe to slippage.

In parts 2 and 3 of our investigation, the tendons were attached to a metal plate (size $70 \times 50 \mathrm{~mm}$, see Figure 4) via sutures woven around wires of about $0.7 \mathrm{~mm}$ in diameter. The wires are laterally plugged in the plate and are accessible via drilled holes of
$4 \mathrm{~mm}$ diameter, which are arranged with two holes in a line perpendicular to the tension direction of the tendon (corresponding to the use of two anchors in the bone, for the $\mathrm{S}$, the $\mathrm{M}$ and the MA stitches) and a third hole staggered (for the application of the D technique). To compensate the compliance of the system tendon-suture joint-metal plate for that of the intact tendon bone complex, the bending of the individual bones were measured in the pre-test. Thus, the contributions of the biomechanical properties of the elements each, tendon and bone, could be separately recorded. When using the metal plate, the contributions of the tendon compliance and that of its natural joint to the bone have to be subtracted from the compliance of the system tendon-suture attachment-metal plate. The compliance of the metal plate is negligible compared with that of the tendon and/or the bending of the bone. According to our test strategy, results obtained with that alternative method should be comparable with those using the bone-tendon complex. The comparison of the stabilities of the tendon attachments obtained with the two test-modes, respectively, was left to the statistical analysis.

In part 3, the tendon samples have been mounted to the metal plate with a reduced number of sutures as described above. According to the reduced stability of tendon fixation, the prescribed cycle number was chosen with a value of 1000 instead of 2500 with the double loaded two-anchor system. The motivation for performing these tests was to compare two different methods to measure gap formation. The cycle numbers to reach a gap of 4 and $5 \mathrm{~mm}$ were counted from the video records made compared with the electronic measurement of gap formation.

\section{Evaluation of data}

The relationship $\delta$ of the displacement peaks corresponding to the upper load levels $(180 \mathrm{~N})$ with the cycle number $n$ shows a creep-like behaviour:

$\delta(n)=a+b[1+n /(\tau . \lambda)]^{-\lambda}$

Parameter $a$ denotes the final value of the displacement because of load cycling, $b$ the extent of resilience, $\tau$ a time constant and $\lambda$ a parameter which accounts for the shape of the relationship displacements - load cycle number. A pure exponential function will result when $\lambda$ approaches infinite. Each measured curve, i.e. the curve recruited from the upper levels of $180 \mathrm{~N}$, was fitted with that function. The curve resulting from the pre-test was then shifted by the pre-defined gap levels of 3,4 and $5 \mathrm{~mm}$, and 


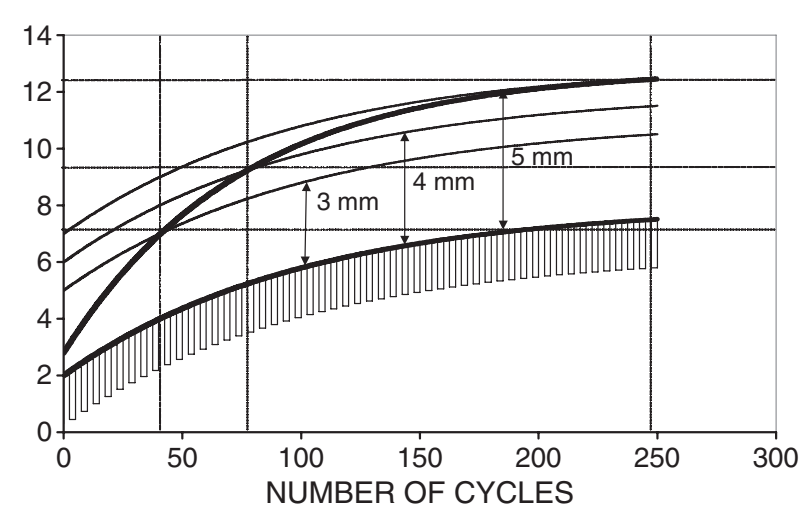

Figure 5: Displacement peak corresponding to the upper load level of $180 \mathrm{~N}$ of the load cycles and the fit with function $\delta$ [Equation (1)]: line for the pre-test illustrated together with response to load cycles and thick line for the test after suture application. The line for the pre-test was shifted about 3, 4 and $5 \mathrm{~mm}$ and the intersection with the thick line (indicated by the dashed gridlines) gives the number $n$ of load cycles to achieve gap formations of $\delta=3,4$ and $5 \mathrm{~mm}$, respectively. The origin of the plot was taken at an initial load of $10 \mathrm{~N}$

the shifted pre-test curve was intersected with the curve obtained with the repaired tendon-bone specimen (Figure 5). The corresponding value of cycle number $n$ is then the cycle number to achieve the gap-level of 3, 4 and $5 \mathrm{~mm}$. The origin of the coordinate system was chosen at the displacement level of the initial load of $10 \mathrm{~N}$. This offset was chosen according to surgical practice.

\section{Statistical analysis}

A three-way analysis of variances was employed for the first run. Factor A was the suturing technique, factor $\mathrm{B}$ the test-modes (bone-tendon preparations and metal plate) and factor $C$ the level of compliance (3, 4 and $5 \mathrm{~mm})$. The significances of factors $A$ and (of course C) were $\geq 99.9 \%$, factor B (test modes) was not significant $(\sim 80 \%)$. A two-way analysis of variances was used in part 3 with factor A (suturing technique) and factor C (gap level). The significance of factor A was $\geq 99 \%$, that of factor C $\geq 99.9 \%$. The correlation between the electronic and optical measurement method was performed via the Pearson coefficient.

\section{Experimental Results}

Throughout the study, results are expressed as mean (SEM) values. With reference to the pre-tests $(n=88)$, the overall final value of the compliance of the tendon-bone complex was mean 5.0 (0.2) $\mathrm{mm}$ after 250 load cycles. The respective overall final value for the tendons alone was mean $3.2(0.2) \mathrm{mm}$.

The values (compensated for an initial load of $10 \mathrm{~N}$ while tendon fixation) of cycle numbers to reach a chosen level of gap formation (specimens of part 1 and 2 of the investigation grouped together) because of slackening of the suture are shown in Table 1 . The $\mathrm{D}$ technique is the most stable one with the lowest values of compliance at all selected levels of gap formation (3, 4 and $5 \mathrm{~mm}$ ). The $\mathrm{M}$ suture configuration shows comparable cycle numbers when

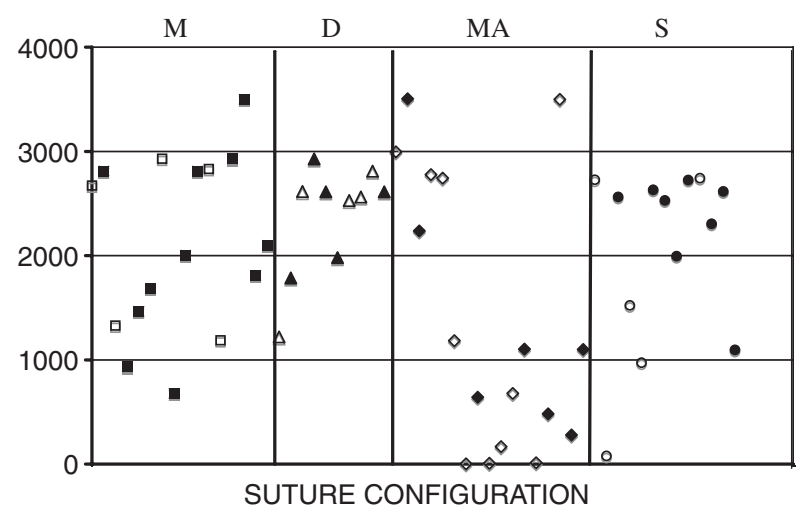

Figure 6: Results obtained with the tendon-anchor-bone system compared with the method to fix the tendon to the metal plate ( mattress, $\bullet$ double row, $\bullet$ modified Mason-Allen and

- single stitches; full symbols: bone; open symbols: metal plate)

\begin{tabular}{|c|c|c|c|c|c|}
\hline Sutures & Test mode & $n$ & $\delta=3 \mathrm{~mm}$ & $\delta=4 \mathrm{~mm}$ & $\delta=5 \mathrm{~mm}$ \\
\hline \multirow[t]{3}{*}{$M$} & Bone-tendon & II & $404(285)$ & $1027(128)$ & $2066(264)$ \\
\hline & Metal plate & 5 & $237(95)$ & $1039(183)$ & $2188(383)$ \\
\hline & Both test modes & 16 & $358(178)$ & $103 \mid(101)$ & $2104(2 \mid I)$ \\
\hline \multirow[t]{3}{*}{ MA } & Bone-tendon & 7 & $443(380)$ & $668(217)$ & 1335 (435) \\
\hline & Metal plate & 10 & $149(74)$ & $689(226)$ & I407 (454) \\
\hline & Both test modes & 17 & $270(160)$ & $680(155)$ & $1377(3 \mid 2)$ \\
\hline \multirow[t]{3}{*}{ S } & Bone-tendon & 8 & $354(I 12)$ & I I 28 (I08) & $2308(192)$ \\
\hline & Metal plate & 5 & $189(99)$ & $799(261)$ & $1609(515)$ \\
\hline & Both test modes & 13 & 291 (79) & $100 \mid(123)$ & 2039 (238) \\
\hline \multirow[t]{3}{*}{$D$} & Bone-tendon & 5 & $924(160)$ & $1123(113)$ & $2384(2 \mid 4)$ \\
\hline & Metal plate & 5 & II8 (69) & 1190 (139) & $2348(286)$ \\
\hline & Both test modes & 10 & $521(157)$ & II56 (85) & $2366(169)$ \\
\hline
\end{tabular}

Table I: Number of cycles necessary to achieve different levels of gap formation $(3,4$ and $5 \mathrm{~mm}) .4$ or 6 sutures were used for the M, MA, and the $\mathrm{S}$ technique or the $\mathrm{D}$ technique, respectively. Results were referenced to a load of $10 \mathrm{~N}$ (the lower load plateau). The values shown are compensated for the compliances of the bone and/or tendons themselves. Results are expressed as mean (SEM) values 
Table 2: Number of cycles necessary to achieve different levels of gap formation ( 3,4 and 5 mm). 2 sutures were used. Results were referenced to a load of $10 \mathrm{~N}$ (the lower load plateau). The values shown are compensated for the compliances of the bone and tendons themselves. The last column shows the results obtained with the measurements using markers and a video camera. Results are expressed as mean (SEM) value

\begin{tabular}{|c|c|c|c|c|c|}
\hline Sutures & $n$ & $\delta=3 \mathrm{~mm}$ & $\delta=4 \mathrm{~mm}$ & $\delta=5 \mathrm{~mm}$ & $\delta=5 \mathrm{~mm}$ (video) \\
\hline$M$ & 10 & $5.4(1.6)$ & $26.5(11.7)$ & $75.8(18.9)$ & $74.5(20.1)$ \\
\hline MA & 12 & $3.5(0.8)$ & $9.3(2.6)$ & $28.1(11.9)$ & $30.2(10.4)$ \\
\hline$S$ & 10 & $3.2(0.7)$ & $7.3(2.4)$ & | 4.0 (4.8) & I3.4 (3.0) \\
\hline
\end{tabular}

compared with the S configuration. The most compliant technique was the modified MA configuration for the gap levels of 4 and $5 \mathrm{~mm}$. At the gap level of $3 \mathrm{~mm}$, the MA technique equals the $\mathrm{S}$ technique (within experimental error). Continuing load-cycling obviously weakens the MA repairs to a greater extent when compared with the $\mathrm{M}$ or $\mathrm{S}$ technique.

As opposed to other studies [29], a gap formation of $10 \mathrm{~mm}$ was not reached within the prescribed 2500 load cycles. In other words, all specimens cycled out. This observation confirms the stability of the fixation method (with two or three double loaded anchors) in principle.

After checking that the results of gap formation with increasing cycle numbers do not depend on the test mode (bone-tendon complex versus metal plate, Figure 6), another set of samples were pre-tested for part 3 of the investigation, the tendons detached from the bone and mounted on the metal plate. The average relationship of gap formation (as measured by means of the position gauge of the testing device together with the above described corrections) with the cycle numbers needed is shown in Table 2 . The cycle numbers have also been counted from the video records made. In part 3 with a reduced number of suture threads for tendon fixation, the $S$ stitches reveal to be the most compliant ones, the modified MA technique shows intermediate values, and the $M$ was most stable. A good agreement between the electronic and video based measurement was found $(r=98.6 \%)$.

\section{Discussion}

When performing the tests, we have to keep in mind that there is always a conflict between the strategies having a pure material test that may be carried out under conditions far away from the natural situation (e.g. testing the suture alone, testing the anchor alone) or on the other hand, testing a structured system, thus simulating closely the natural situation with several inherent influences. These influences often cannot be controlled in such a way that the properties of a single component of a system can be registered irrespective of the effect of interactions with the other system components.

In this study, the goal was to determine the differences of biomechanical stability of several fixation techniques of tendon to bone. The system tested may be seen as composed of tendon, tendon attachment to bone and bone. One very important point is that for suture testing the load applied at the free end of the tendon has to be transmitted completely to the anchor-suture system. Therefore, we cut off the tendon from the bone. In the contrary, a partial defect (although the most frequently occurring one) would result in a subdivision of the applied load into portions carried by the remaining natural joint between tendon and bone and the joint given by the sutureanchor system. However, these load portions remain unknown if not separately measured.

With reference to the goal of the study, we have to work out the stability of the tendon-bone joint only, thus separating the biomechanical quality of bone and tendon themselves, respectively. This should be accomplished by performing the pre-tests under the chosen test conditions. It might be argued that the biomechanical properties may be affected by a repeatedly loading of the samples. However, there was a time interval of about $4 \mathrm{~h}$ between pre-test and test. Thus, according to this 'long' period of rest the original biomechanical properties should have been recovered, insofar as the applied maximum of load of $180 \mathrm{~N}$ is as small as to produce no irreversible damage to the intact tendon or bone (e.g. [33, 34]). The advantage of this method of pre-testing is that the elastic property of each specific tendon is recorded. This property is then subtracted from the test results of the tendon-suture-bone complex. This method is the most accurate one $(2 \mu \mathrm{m})$ with as few external influences as possible. It allows the measurement of the mechanical quality of the suture fixation without an external device such as calipers or metric rulers.

The cycle numbers to achieve the chosen gaps of 3 , 4 and $5 \mathrm{~mm}$ are markedly reduced after the number of sutures was halved (compare Tables 1 and 2). The load on the sutures is then doubled compared with 
the tests with four sutures (two double-loaded anchors). With these tests, the agreement of gap formation measured with the internal position measurement device and with the video camera was excellent $(r=98.6 \%)$.

\section{Conclusions}

For the surgeon, the results of this study confirm also in line with other authors that the double row technique is the most stable one. The modified Mason-Allen and mattress suture configuration are performing nearly equally but suturing four mattress stitches especially in an arthroscopic way is not so demanding to the surgeon.

With regard to the rehabilitation protocol, a strong repair is important. So therefore the surgeon should use whenever it is possible and no double-row repair is performed at least two anchors in a row and four sutures because of the much better stability as the experiment and the mechanical analysis suggest.

One very important thing to stress is that all suture techniques cycled out 2500 cycles as it is crucial for every surgeon to know that the fixation technique used is stable and does not tear on carefully assisted mobilization in slight movements. And it is also necessary to know that a trauma or promptly load leads to early failure of any suture configuration or rupture of the anchor suture eyelet. We conducted parts 2 and 3 in the investigation to study also a possible effect of the compliance of the anchor eyelet on the stability of the suture systems. Attaching the tendons to the metal plate via sutures woven around the wires might result in an altered compliance of the systems. This was not observed in the study.

\section{REFERENCES}

1. Reilly, P., Macleod, I., Macfarlane, R., Windley, J. and Emery, R. J. (2006) Dead men and radiologists don't lie: a review of cadaveric and radiological studies of rotator cuff tear prevalence. Ann. R. Coll. Surg. Engl. 88, 116-121.

2. Tempelhof, S., Rupp, S. and Seil, R. (1999) Age-related prevalence of rotator cuff tears in asymptomatic shoulders. J. Shoulder Elbow Surg. 8, 296-299.

3. Fuchs, S., Chylarecki, C. and Langenbrinck, A. (1999) Prevalence and symptoms of clinically manifest rotator cuff lesions. Int. J. Sports Med. 20, 201-205.

4. Jones, A. O. (1998) Magnetic resonance imaging of the supraspinatus tendon: the significance of signal intensity alterations at the 'critical zone'. Aust. Radiol. 42, 106113.

5. Milgrom, C., Schaffler, M., Gilbert, S. and van-Holsbeeck, M. (1995) Rotator-cuff changes in asymptomatic adults: the effect of age, hand dominance and gender. J. Bone Joint Surg. Br. 77, 296-298.
6. Huijsmans, P. E., Pritchard, M. P., Berghs, B. M., van Rooyen, K. S., Wallace, A. L. and de Beer, J. F. (2007) Arthroscopic rotator cuff repair with double-row fixation. J. Bone Joint Surg. Am. 89, 1248-1257.

7. Sugaya, H., Maeda, K., Matsuki, K. and Moriishi, J. (2007) Repair integrity and functional outcome after arthroscopic double-row rotator cuff repair: a prospective outcome study. J. Bone Joint Surg. Am. 89, 953-960.

8. Cole, B. J., McCarty, L. P., III, Kang, R. W., Alford, W., Lewis, P. B. and Hayden, J. K. (2007) Arthroscopic rotator cuff repair: prospective functional outcome and repair integrity at minimum 2-year follow-up. J. Shoulder Elbow Surg. 16, 579-585 (epub 12 July 2007).

9. Boileau, P., Brassart, N., Watkinson, D. J., Carles, M., Hatzidakis, A. M. and Krishnan, S. G. (2005) Arthroscopic repair of full-thickness tears of the supraspinatus: does the tendon really heal? J. Bone Joint Surg. Am. 87, 1229-1240.

10. Galatz, L. M., Ball, C. M., Teefey, S. A., Middleton, W. D. and Yamaguchi, K. (2004) The outcome and repair integrity of completely arthroscopically repaired large and massive rotatorcuff tears. J. Bone Joint Surg. Am. 86, 219224.

11. Gazielly, D. F., Gleyze, P. and Montagnon, C. (1994) Functional and anatomical results after rotator cuff repair. Clin. Orthop. Relat. Res. 304, 43-53.

12. Harryman, D. T., Mack, L. A., Wang, K. Y., Jackins, S. E., Richardson, M. L. and Matsen, F. A. (1991) Repairs of the rotator cuff correlation of functional results with integrity of the cuff. J. Bone Joint Surg. Am. 73, 982-989.

13. Gerber, C., Schneeberger, A. G., Beck, M. and Schlegel, U. (1994) Mechanical strength of repairs of the rotator cuff. J. Bone Joint Surg. 76-B, 371-380.

14. Cummins, C. A. and Murrell, G. A. (2003) Mode of failure for rotator cuff repair with suture anchors identified at revision surgery. J. Shoulder Elbow Surg. 12, 128-133.

15. Goradia, V. K., Mullen, D. J., Boucher, H. R., Parks, B. G. and O'Donnell, J. B. (2001) Cyclic loading of rotator cuff repairs: a comparison of bioabsorbable tacks with metal suture anchors and transosseous sutures. Arthroscopy 17, 360-364.

16. Burkhart, S. S., Johnson, T. C., Wirth, M. A. and Athanasiou, K. A. (1997) Cyclic loading of transosseous rotator cuff repairs: tension overload as a possible cause of failure. Arthroscopy 13, 172-176.

17. Kim, D. H., Elattrache, N. S., Tibone, J. E., Jun, B. J., DeLaMora, S. N., Kvitne, R. S. and Lee, T. Q. (2005) Biomechanical comparison of a single-row versus double-row suture anchor technique for rotator cuff repair. Am. J. Sports Med. 34, 407-414 (epub 10 November 2005).

18. Ma, C. B., Comerford, L., Wilson, J. and Puttlitz, C. M. (2006) Biomechanical evaluation of arthroscopic rotator cuff repairs: double-row compared with single-row fixation. J. Bone Joint Surg. Am. 88, 403-410.

19. Smith, C. D., Alexander, S., Hill, A. M., Huijsmans, P. E., Bull, A. M., Amis, A. A., DeBeer, J. F. and Wallace, A. L. A. (2006) A biomechanical comparison of single and doublerow fixation in arthroscopic rotator cuff repair. J. Bone Joint Surg. Am. 88, 2425-2431.

20. Mazzocca, A. D., Millett, P. J., Guanche, C. A., Santangelo, S. A. and Arciero, R. A. (2005) Arthroscopic single-row 
versus double-row suture anchor rotator cuff repair. Am. J. Sports Med. 33, 1861-1868 (epub 6 October 2005).

21. De Carli, A., Vadalà, A., Monaco, E., Labianca, L., Zanzotto, E. and Ferretti, A. (2005) Effect of cyclic loading on new polyblend suture coupled with different anchors. Am. J. Sports Med. 33, 214-219.

22. Craft, D. V., Moseley, J. B., Cawley, P. W. and Noble, P. C. (1996) Fixation strength of rotator cuff repairs with suture anchors and the transosseous suture technique. J. Shoulder Elbow Surg. 5, 32-40.

23. Koganti, A. K., Adamson, G. J., Gregersen, C. S., Pink, M. M. and Shankwiler, J. A. (2006) Biomechanical comparison of traditional and locked suture configurations for arthroscopic repairs of the rotator cuff. Am. J. Sports Med. 34, 1832-1838 (epub 21 July 2006).

24. Petit, C. J., Boswell, R., Mahar, A., Tasto, J. and Pedowitz, R. A. (2003) Biomechanical evaluation of a new technique for rotator cuff repair. Am. J. Sports Med. 31, 849-853.

25. Mahar, A., Tamborlane, J., Oka, R., Esch, J. and Pedowitz, R. A. (2007) Single-row suture anchor repair of the rotator cuff is biomechanically equivalent to double-row repair in a bovine model. Arthroscopy 23, 1265-1270. (epub 7 November 2007).

26. Milano, G., Grasso, A., Zarelli, D., Deriu, L., Cillo, M. and Fabbriciani, C. (2008) Comparison between single-row and double-row rotator cuff repair: a biomechanical study. Knee Surg. Sports Traumatol. Arthrosc. 16, 75-80.

27. Klinger, H. M., Buchhorn, G. H., Heidrich, G., Kahl, E. and Baums, M. H. (2007) Biomechanical evaluation of rotator cuff repairs in a sheep model: suture anchors using arthroscopic Mason-Allen stitches compared with transosseous sutures using traditional modified Mason-Allen stitches. Clin. Biomech. (Bristol, Avon) 30, 00-00 (epub ahead of print).

28. Reardon, D. J. and Maffulli, N. (2007) Clinical evidence shows no difference between single- and double-row repair for rotator cuff tears. Arthroscopy 23, 670-673.

29. Ma, C. B., MacGillivray, J. D., Clabeaux, J., Lee, S. and Otis, J. C. (2004) Biomechanical evaluation of arthroscopic rotator cuff stitches. J. Bone Joint Surg. Am. 86, 1211-1216.

30. Scheibel, M. T. and Habermeyer, P. (2003) A modified Mason-Allen technique for rotator cuff repair using suture anchors. Arthroscopy 19, 330-333.

31. De Beer, J. F., van Rooyen, K. and Boezaart, A. P. (1998) Nicky's knot - a new slip knot for arthroscopic surgery. Arthroscopy 14, 109-110.

32. Wetzler, M. J., Bartolozzi, A. R., Gillespie, M. J., Roth, C. A., Ciccotti, M. G., Snyder-Mackler, L. and Santare, M. H. (1996) Fatigue properties of suture anchors in anterior shoulder reconstructions: Mitek GII. Arthroscopy 12, 687693.

33. Fung, Y. C. (1984) Biomechanics, Mechanical Properties of living Tissues. Springer Verlag, Berlin Heidelberg New York.

34. Viidik, A. (1979) Biomechanical behaviour of soft connective tissues. In: Progress in Biomechanics (N. Akkas, Ed.). Sijthoff \& Nordhoff, Alphen aan de Rijn, the Netherlands: 75-113. 